\title{
POSSIBLE RÓLE OF THE WHITE DWARF IN GRAIN FORMATION IN CV SYSTEMS
}

\author{
J. S. Albinson, A. Evans, \\ Department of Physics, University of Keele, \\ Keele, Staffordshire, ST5 5BG, \\ United Kingdom.
}

ABSTRACT. Zhilyaev \& Zubko $^{3}$ described white dwarf model atmospheres in which carbon might condense. Whittaker presented a version of the phase diagram for carbon on which there is a region where carbyne is thermodynamically favoured over graphite. Because the ratio of cross-section to mass is much higher for a thin cylinder, a carbyne cylinder has a much better chance of being blown out of a white dwarf atmosphere by radiation pressure. However even for small grains, gravity overcomes radiation pressure for cylinders, as it does for spherical grains. In the case of a non-polar cataclysmic variable (CV) system, the white dwarf is surrounded by an accretion disk and the luminosity of the disk may provide sufficient additional radiation pressure to drive a grain out of the system. If a grain can initially be transported out of the white dwarf atmosphere there may be several CV systems in which the combined radiation pressure of the disk and white dwarf can blow grains out of the system. For small grains and white dwarf masses $>0.5 \mathrm{M}$ radiation pressure can overcome gravity. These remarks also apply to grains that originate in the cooler regions of the disk, where the density may be high enough to sustain grain formation. Thus some non-polar CV systems may possess circumstellar dust shells, whose composition may reflect the nature of the white dwarf or secondary. For cases in which grains originate in the disk any circumstellar dust may be transient, as the mass transfer varies. Furthermore, in classical nova systems, this process may provide grain precursors, on which larger grains might grow during a noya outburst. A more complete discussion may be found in Albinson \& Evans.

\section{References.}

1. Albinson, J. S. \& Evans, A., 1986, in "Cataclysmic Variables", IAU Colloquium 92, D. Reidel, in press.

2. Whittaker, A. G., 1978, Science, 200, 763.

3. Zhilayev, B. E. \& Zubko, V. G., 1983, Sov. Ast. Lett., 9, 122. 